Journal of Organometallic Chemistry, 376 (1989) 333-342

Elsevier Sequoia S.A., Lausanne - Printed in The Netherlands

JOM 20119

\title{
Unusual tripodal ligands. Synthesis of 2,2-bis(diphenylphosphinomethyl)-1-phenylthiopropane and its group VI complexes
}

\author{
Shiuh-Tzung Liu *, Hsin-Ell Wang, Ming-Chu Cheng and Shie-Ming Peng * \\ Department of Chemistry, National Taiwan University, Taipei, Taiwan I0764 (Republic of China)
}

(Received April 26th, 1989)

\begin{abstract}
A hybrid tripodal ligand containing phosphorus and sulfur atoms, 2,2-bis(diphenylphosphinomethyl)-1-phenylthiopropane $\left(\mathbf{P}_{2} \mathbf{S}\right)$, was synthesized and its metal carbonyl complexes $\left(\mathbf{P}_{\mathbf{2}} \mathbf{S}\right) \mathrm{M}(\mathrm{CO})_{4}(\mathrm{M}=\mathrm{Cr}$, Mo and $\mathrm{W})$ were prepared. All synthetic intermediates and complexes have been characterized by spectroscopic methods and elemental analysis. The spectroscopic identification of complexes shows that the $\mathbf{P}_{2} \mathbf{S}$ ligand behaves as a bidentate ligand with two phosphorus atoms coordinating to the metal center. The structure was confirmed by a crystal structure study of $\left(\mathbf{P}_{2} \mathbf{S}\right) \mathrm{Mo}(\mathrm{CO})_{4}$, which revealed an octahedral coordination around the molybdenum atom, and two cis-coordinated phosphorus donors. The conformation of the six-membered chelate ring is a twisted-boat and the possible reasons for this conformational arrangement are discussed.
\end{abstract}

\section{Introduction}

Over the past decade, the coordination and organometallic chemistry of triphos 1 have gained considerable interest owing to the rigidity of this ligand [1-5]. However, this triphos can function as a tridentate or a bidentate ligand, such as in fac-(triphos) $\mathrm{Cr}(\mathrm{CO})_{3}[4]$ and (triphos) $\mathrm{Pt}\left(\mathrm{P} \equiv \mathrm{C}-\mathrm{Bu}^{t}\right)$ [5]; respectively. As part of our study on the tripodal phosphorus ligands, we have synthesized such species that contain differing donor sites. Such ligands can be used in studies of the coordination behavior toward various metals. Recently we reported the synthesis of a tripodal ligand containing phosphorus and oxygen donors [3], 2,2-bis(diphenylphosphinomethyl)-1-methoxypropane (2). Here we describe our synthesis of another hybrid tripodal ligand which contains phosphorus and sulfur atoms, 2,2-bis(diphen- 
ylphosphinomethyl)-1-phenylthiopropane (3), and its group VI metal carbonyl complexes.

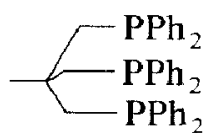

(1)

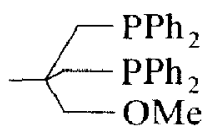

(2)

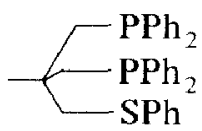

(3)

\section{Results and discussion}

Synthesis of the ligand. The preparation of tripodal ligand 3 is shown in Scheme 1. If triol $\mathbf{4}$ is used as the starting material, the synthesis must involve a differentiation of three hydroxy functions at some point during the synthesis. Triol 4 was transformed into ketal alcohol 5 followed by conversion into tosylate 6 in high yield by previously published procedures [6]. Treatment of 6 with an excess of benzenethiolate in ethanol gives the desired sulfide 7 . Hydrolysis of $\mathbf{7}$ gives $\mathbf{8}$, which is converted into the mesylate 9 . Complex 9 is converted into the desired phosphine by the reaction of 9 with diphenylphosphide anion, generated from the reductive cleavage of triphenylphosphine with lithium metal in THF, followed by anaerobic chromatography. This synthesis gives the desired tripodal ligand in $38 \%$ yield (starting from triol 4). Compound $\mathbf{3}$ has been characterized by spectroscopic methods and elemental analysis. The ${ }^{1} \mathrm{H}$ NMR spectrum shows a doublet absorption at $2.48 \mathrm{ppm}[\mathrm{J}(\mathrm{P}-\mathrm{C}-\mathrm{H}) 3 \mathrm{~Hz}]$ with integration of four protons which indicates that two phosphorus groups are attached to the methylene units. The single absorption at $-25.57 \mathrm{ppm}$ in the ${ }^{31} \mathrm{P}$ NMR spectrum confirms this.

Complexation studies. The metal hexacarbonyls, $\mathrm{M}(\mathrm{CO})_{6}$, were refluxed in aromatic solvents [toluene for $\mathrm{Mo}(\mathrm{CO})_{6}$; xylene for $\mathrm{Cr}(\mathrm{CO})_{6}$ and $\mathrm{W}(\mathrm{CO})_{6}$ ] with an equimolar amount of ligand (3), to give the substituted tetracarbonyl derivatives, (3) $\mathrm{M}(\mathrm{CO})_{4}[10 \mathrm{a} \mathrm{M}=\mathrm{Cr} ; 10 \mathrm{~b} \mathrm{M}=\mathrm{Mo} ; 10 \mathrm{c} \mathrm{M}=\mathrm{W}]$ (eq. 1). All of these reactions were monitored by IR spectroscopy, which showed that $8-10 \mathrm{~h}$ were required for all of metal carbonyls to be converted into tetracarbonyl species. It was found that continued heating or photochemical activation resulted only in the decomposition of (3) $\mathrm{M}(\mathrm{CO})_{4}$ with no indication of formation of $(3) \mathrm{M}(\mathrm{CO})_{3}$. Compounds $10 \mathrm{a}-10 \mathrm{c}$

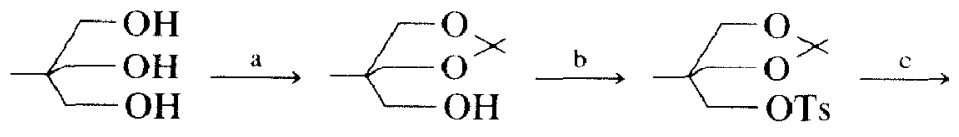

(4)

(5)

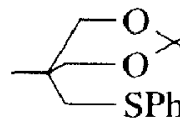

(7)
(6)

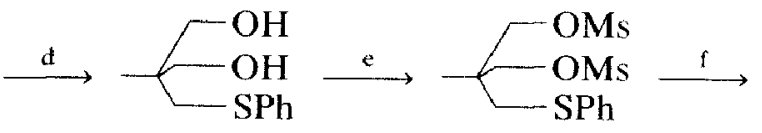

(9)

Scheme 1. a, Acetone/P-TsOH, 94\%. b, TsCl/Py, 98\%. c, PhSNa/EtOH, 88\%. d, Water/EtOH $/ \mathrm{HCl}$. 93\%. e, MsCl/triethylamine, $88 \%$. f, $\mathrm{Ph}_{2} \mathrm{PLi} / \mathrm{THF} 57 \%$. 


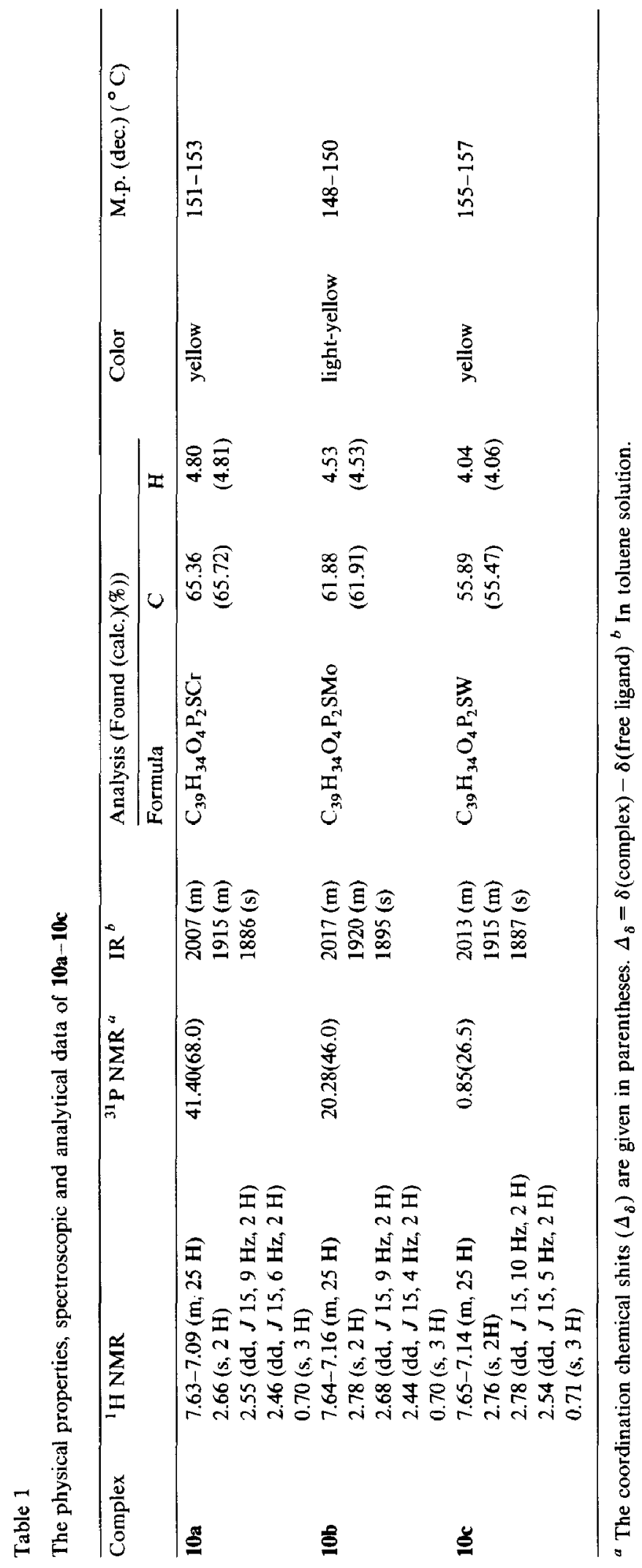


were all purified by chromatography on silica gel. The physical properties and spectroscopic and analytical data are given in Table 1. In the ${ }^{1} \mathrm{H}$ NMR spectrum, the methylene units attached to phosphorus appear as an ABX pattern, which results from the geminal coupling $(J 15 \mathrm{~Hz})$ of two hydrogen atoms and the splitting by phosphorus atom $[J(\mathrm{P}-\mathrm{C}-\mathrm{H})]$. The tendency to undergo coordination chemical shifts decreases as $\mathrm{Cr}>\mathrm{Mo}>\mathrm{W}$, which is similar to that of $(\mathrm{dppp}) \mathrm{M}(\mathrm{CO})_{4}$ [where dppp $=\mathrm{Ph}_{2} \mathrm{PCH}_{2} \mathrm{CH}_{2} \mathrm{CH}_{2} \mathrm{PPh}_{2}$ ] [7].

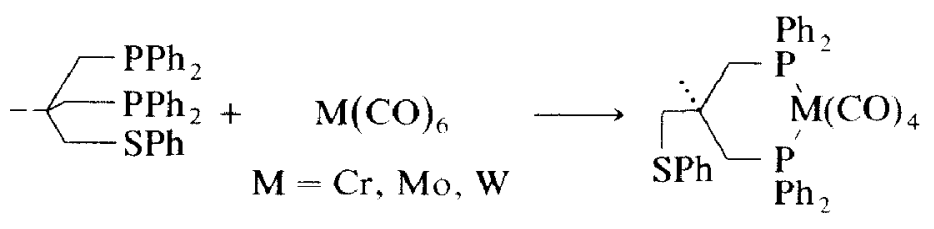

$$
\begin{aligned}
& (10 \mathrm{a}: \mathrm{M}=\mathrm{Cr} ; \\
& 10 \mathbf{b}: \mathrm{M}=\mathrm{Mo} ; \\
& 10 \mathrm{c}: \mathrm{M}=\mathrm{W})
\end{aligned}
$$

Crystals of complex 10b were isolated from ethyl acetate and hexane. The ORTEP plot of 10b is depicted in Fig. 1. Selected bond distances and bond angles are listed in Table 2. The molybdenum center is surrounded by two cis-coordinated phosphorus donors and four carbonyls, so that the metal center has octahedral geometry. Although both phosphorus-molybdenum distances are in the usual range of 2.52(1) and 2.54(1) $\AA$, their difference $(0.02 \AA)$ is slightly larger than that of $\left(\mathbf{P}_{2} \mathbf{O}\right) \mathrm{Mo}(\mathrm{CO})_{4}(\mathbf{1 1})^{*}$. The $\mathrm{P} 1-\mathrm{Mo1}-\mathrm{P} 2$ interligand angle of $85.3(4)^{\circ}$ is similar to that in 11. The difference $(0.2 \AA)$ of two metal-carbon bonds trans to the phosphorus atoms, we think is due to the poor quality of the intensity data ( $R=0.075$ ), and not to actual structural differences.<smiles>COCC1(C)C[PH](c2ccccc2)C(C(=O)O[Na])(c2ccccc2)C1</smiles>

(11)

Nevertheless, this crystal study does confirm the structure of $\mathbf{1 0 b}$, which also reveals the twisted-boat conformation for the six-membered chelate ring. This particular conformation of the Mo-P1-C1-C2-C3-P2 ring is confirmed by the characteristic $+g,-g$ alternations (Table 3 ). Theoretical calculations by Gollogly and Hawkins [8] show that the chair conformation would be the most stable form in six-membered chelate rings associated with the octahedral complex, and the complex $(\mathrm{OC})_{4} \mathrm{WPPh}_{2} \mathrm{CH}\left(\mathrm{CH}_{2} \mathrm{CH}_{2} \mathrm{PPh}_{2}\right) \mathrm{CH}_{2} \mathrm{PPh}_{2}$ does have a six-membered chelate

\footnotetext{
* Unpublished results. Molecular structure of 11 has been determined. Mo-P bond lengths are 2.519(1)
} and $2.516(1) \AA$; P-Mo-P bond angle $85.08(4)^{\circ}$. 


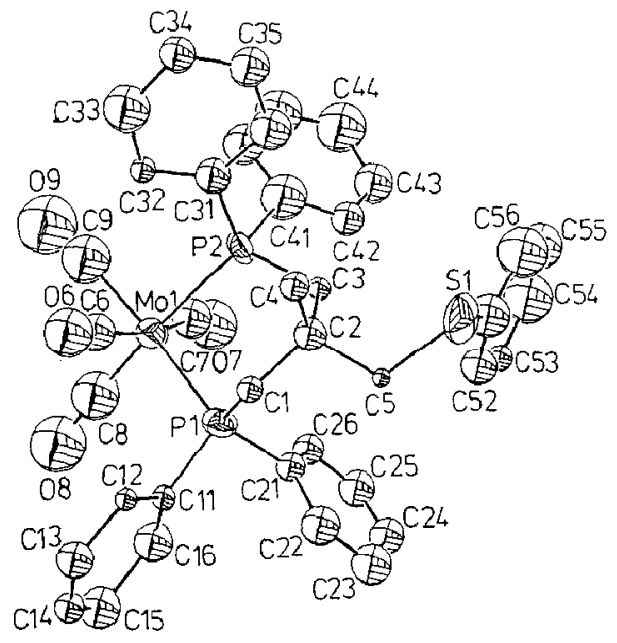

Fig. 1. The ORTEP plot of complex 10b

ring in the chair form with the $\mathrm{Ph}_{2} \mathrm{PCH}_{2}$ substituent occupying an equatorial position [9]. In our complex, 10b, the six-membered chelate ring prefers the twisted-boat form and the reason for this conformational arrangement arises from the relief of the strained 1,3-diaxial interaction between the phenyl group and the

Table 2

Selected bond distances $(\AA)$ and bond angles $\left({ }^{\circ}\right)$ in $\mathbf{1 0 b}$

\begin{tabular}{lrlr}
\hline Mo-P1 & $2.52(1)$ & Mo-P2 & $2.54(1)$ \\
Mo-C6 & $1.97(4)$ & Mo-C7 & $1.99(4)$ \\
Mo-C8 & $1.82(5)$ & Mo-C9 & $2.08(5)$ \\
P1-C1 & $1.87(3)$ & $\mathrm{C} 1-\mathrm{C} 2$ & $1.52(5)$ \\
P2-C3 & $1.90(3)$ & $\mathrm{C} 2-\mathrm{C} 3$ & $1.60(5)$ \\
C6-O6 & $1.14(5)$ & $\mathrm{C} 7-\mathrm{O} 7$ & $1.27(6)$ \\
C8-O8 & $1.29(6)$ & $\mathrm{C} 9-\mathrm{O} 9$ & $1.11(6)$ \\
P1-Mo-P2 & $85.3(4)$ & P1-Mo-C6 & $84(1)$ \\
P1-Mo-C7 & $107(1)$ & P1-Mo-C8 & $93(2)$ \\
P1-Mo-C9 & $169(2)$ & P2-Mo-C6 & $96(1)$ \\
P2-Mo-C7 & $85(1)$ & P2-Mo-C8 & $174(2)$ \\
P2-Mo-C9 & $95(1)$ & Mo-P1-C1 & $110(1)$ \\
P1-C1-C2 & $122(2)$ & $\mathrm{C} 1-\mathrm{C} 2-\mathrm{C} 3$ & $114(3)$ \\
C2-C3-P2 & $119(2)$ & & \\
\hline
\end{tabular}

Table 3

Torsional angles $\left({ }^{\circ}\right)$ of the six-membered ring, Mo-P1-C1-C2-C3-P2

\begin{tabular}{lr}
\hline Mo-P1-C1-C2 & $-73(2)$ \\
P1-C1-C2-C3 & $22(1)$ \\
C1-C2-C3-P2 & $51(2)$ \\
C2-C3-P2-Mo & $-62(2)$ \\
C3-P2-Mo-P1 & $7(1)$ \\
P2-Mo-P1-C1 & $43(1)$ \\
\hline
\end{tabular}


Table 4

The atomic coordinates and thermal parameters

\begin{tabular}{|c|c|c|c|c|}
\hline & $x$ & $y$ & $z$ & $B_{1 \mathrm{No}}$ \\
\hline Mol & $0.21093(18)$ & $0.36270(19)$ & 0.09087 & $3.84(20)$ \\
\hline $\mathrm{Mo} 2$ & $0.69312(18)$ & $0.34109(18)$ & $0.31579(24)$ & $3.30(19)$ \\
\hline $\mathrm{P} 1$ & $0.2923(5)$ & $0.2632(6)$ & $0.0793(6)$ & $3.3(6)$ \\
\hline $\mathrm{P} 2$ & $0.1340(5)$ & $0.2884(6)$ & $0.0137(6)$ & $3.3(6)$ \\
\hline $\mathrm{P} 3$ & $0.6219(5)$ & $0.2965(6)$ & $0.4188(6)$ & $2.6(6)$ \\
\hline P4 & $0.7862(5)$ & $0.2691(5)$ & $0.3686(6)$ & $2.7(6)$ \\
\hline $\mathrm{S} 1$ & $0.1872(7)$ & $0.0462(6)$ & $-0.0555(7)$ & $6.2(8)$ \\
\hline $\mathrm{S} 2$ & $0.6851(6)$ & $0.0707(6)$ & $0.5327(7)$ & $5.1(7)$ \\
\hline $\mathrm{Cl}$ & $0.2931(16)$ & $0.2301(17)$ & $-0.0151(18)$ & $2.0(8)$ \\
\hline $\mathrm{C} 2$ & $0.2355(18)$ & $0.1872(20)$ & $-0.0451(20)$ & $3.3(10)$ \\
\hline C3 & $0.1667(16)$ & $0.1970(17)$ & $-0.0032(18)$ & $1.5(8)$ \\
\hline $\mathrm{C} 4$ & $0.2295(17)$ & $0.2046(18)$ & $-0.1260(19)$ & $2.4(9)$ \\
\hline $\mathrm{C} 5$ & $0.2538(15)$ & $0.1085(17)$ & $-0.0260(18)$ & $1.1(8)$ \\
\hline C6 & $0.2611(20)$ & $0.4028(22)$ & $0.0098(22)$ & $4.6(12)$ \\
\hline 06 & $0.2908(15)$ & $0.4195(16)$ & $-0.0394(17)$ & $6.9(9)$ \\
\hline $\mathrm{C} 7$ & $0.1487(22)$ & $0.3394(23)$ & $0.1706(24)$ & $5.5(12)$ \\
\hline 07 & $0.1216(17)$ & $0.3257(17)$ & $0.2308(18)$ & $8.5(10)$ \\
\hline $\mathrm{C} 8$ & $0.265(3)$ & $0.409(3)$ & $0.154(3)$ & $7.9(16)$ \\
\hline 08 & $0.3076(19)$ & $0.4383(18)$ & $0.1958(20)$ & $10.1(11)$ \\
\hline $\mathrm{CP}$ & $0.1533(23)$ & $0.4524(24)$ & $0.082(3)$ & $7.8(14)$ \\
\hline 09 & $0.1275(17)$ & $0.5032(19)$ & $0.0788(23)$ & $11.7(12)$ \\
\hline C11 & $0.3791(15)$ & $0.2950(16)$ & $0.0864(20)$ & $1.7(7)$ \\
\hline $\mathrm{C} 12$ & $0.4793(18)$ & $0.3533(21)$ & $0.0397(21)$ & $4.1(10)$ \\
\hline $\mathrm{C} 13$ & $0.4131(15)$ & $0.3216(17)$ & $0.0278(18)$ & $1.5(8)$ \\
\hline $\mathrm{C} 14$ & $0.5016(17)$ & $0.3556(18)$ & $0.1090(18)$ & $2.8(9)$ \\
\hline $\mathrm{C} 15$ & $0.4730(21)$ & $0.3353(23)$ & $0.1674(23)$ & $5.5(12)$ \\
\hline C16 & $0.3997(22)$ & $0.3014(23)$ & $0.1551(23)$ & $5.0(12)$ \\
\hline $\mathrm{C} 21$ & $0.2946(18)$ & $0.1881(18)$ & $0.1402(18)$ & $2.7(9)$ \\
\hline $\mathrm{C} 22$ & $0.3492(20)$ & $0.1401(22)$ & $0.1295(22)$ & $4.8(11)$ \\
\hline$C 23$ & $0.3567(22)$ & $0.0826(22)$ & $0.1772(24)$ & $5.3(12)$ \\
\hline $\mathrm{C} 24$ & $0.3024(20)$ & $0.0800(21)$ & $0.2311(21)$ & $4.2(11)$ \\
\hline$C .25$ & $0.2535(19)$ & $0.1267(22)$ & $0.2490(22)$ & $4.6(11)$ \\
\hline $\mathrm{C} 26$ & $0.2471(19)$ & $0.1831(20)$ & $0.1968(21)$ & $3.3(10)$ \\
\hline C31 & $0.1077(19)$ & $0.3248(19)$ & $-0.0706(21)$ & $3.8(11)$ \\
\hline $\mathrm{C} 32$ & $0.1254(17)$ & $0.3937(17)$ & $-0.0834(19)$ & $2.1(9)$ \\
\hline $\mathrm{C} 33$ & $0.1010(23)$ & $0.4157(25)$ & $-0.1528(24)$ & $6.5(14)$ \\
\hline C34 & $0.0607(18)$ & $0.3739(20)$ & $-0.2039(21)$ & $3.8(11)$ \\
\hline C 35 & $0.0454(19)$ & $0.3046(20)$ & $-0.1841(25)$ & $4.6(11)$ \\
\hline $\mathrm{C} 36$ & $0.0704(21)$ & $0.2762(22)$ & $-0.1188(23)$ & $4.9(12)$ \\
\hline C41 & $0.0485(21)$ & $0.2686(24)$ & $0.054(3)$ & $6.5(14)$ \\
\hline $\mathrm{C} 42$ & $0.0361(18)$ & $0.2037(19)$ & $0.0931(24)$ & $3.6(9)$ \\
\hline $\mathrm{C} 43$ & $-0.0218(21)$ & $0.1913(23)$ & $0.1142(23)$ & $5.4(13)$ \\
\hline $\mathrm{C} 44$ & $-0.0751(24)$ & $0.242(3)$ & $0.114(3)$ & $8.2(16)$ \\
\hline $\mathrm{C} 45$ & $-0.0608(22)$ & $0.3064(24)$ & $0.088(3)$ & $7.0(13)$ \\
\hline C46 & $0.0014(22)$ & $0.3252(22)$ & $0.0526(23)$ & $5.9(12)$ \\
\hline $\mathrm{C} 51$ & $0.1409(21)$ & $0.0265(22)$ & $0.0195(24)$ & $5.7(13)$ \\
\hline $\mathrm{C} 52$ & $0.1647(18)$ & $0.0261(19)$ & $0.0876(25)$ & $4.2(10)$ \\
\hline C53 & $0.1228(18)$ & $0.0118(18)$ & $0.1448(19)$ & $2.4(9)$ \\
\hline C54 & $0.055(3)$ & $-0.001(3)$ & $0.133(3)$ & $7.7(16)$ \\
\hline C55 & $0.0299(21)$ & $0.000(3)$ & $0.068(3)$ & $7.2(15)$ \\
\hline $\mathrm{C} 56$ & $0.0732(25)$ & $0.010(3)$ & $0.002(3)$ & $7.8(16)$ \\
\hline C1A & $0.7721(16)$ & $0.2619(19)$ & $0.4677(19)$ & $2.2(9)$ \\
\hline $\mathrm{C} 2 \mathrm{~A}$ & $0.7225(16)$ & $0.2136(17)$ & $0.4986(17)$ & $1.3(8)$ \\
\hline
\end{tabular}


Table 4 (continued)

\begin{tabular}{|c|c|c|c|c|}
\hline & $x$ & $y$ & $z$ & $B_{\text {iso }}$ \\
\hline $\mathrm{C} 3 \mathrm{~A}$ & $0.6492(16)$ & $0.2108(16)$ & $0.4587(17)$ & $1.2(8)$ \\
\hline C4A & $0.7117(18)$ & $0.2396(21)$ & $0.5778(22)$ & $4.6(10)$ \\
\hline C5A & $0.7455(16)$ & $0.1339(19)$ & $0.4998(18)$ & $2.4(9)$ \\
\hline C6A & $0.6465(20)$ & $0.2685(21)$ & $0.2562(22)$ & $4.4(11)$ \\
\hline O6A & $0.6204(14)$ & $0.2269(14)$ & $0.2223(15)$ & $5.4(8)$ \\
\hline C7A & $0.7422(19)$ & $0.4182(21)$ & $0.3611(22)$ & $4.3(11)$ \\
\hline O7A & $0.7694(15)$ & $0.4664(17)$ & $0.3901(17)$ & $7.4(9)$ \\
\hline C8A & $0.6185(24)$ & $0.406(3)$ & $0.287(3)$ & $7.6(15)$ \\
\hline O8A & $0.5836(17)$ & $0.4450(19)$ & $0.2718(20)$ & $9.7(11)$ \\
\hline C9A & $0.7488(20)$ & $0.3701(23)$ & $0.2321(22)$ & $4.8(11)$ \\
\hline O9A & $0.7775(17)$ & $0.3773(18)$ & $0.1799(18)$ & $8.5(10)$ \\
\hline C11A & $0.5992(18)$ & $0.3465(20)$ & $0.4983(20)$ & $3.4(10)$ \\
\hline $\mathrm{C} 12 \mathrm{~A}$ & $0.5494(21)$ & $0.3209(22)$ & $0.5458(24)$ & $5.4(12)$ \\
\hline C13A & $0.5294(18)$ & $0.3673(21)$ & $0.6074(22)$ & 4.4(11) \\
\hline $\mathrm{C} 14 \mathrm{\Lambda}$ & $0.5578(23)$ & $0.4309(24)$ & $0.612(3)$ & $6.4(14)$ \\
\hline C15A & $0.6002(21)$ & $0.4561(22)$ & $0.5619(23)$ & $5.5(13)$ \\
\hline C16A & $0.6216(19)$ & $0.4202(19)$ & $0.4974(20)$ & $3.3(10)$ \\
\hline $\mathrm{C} 21 \mathrm{~A}$ & $0.5372(17)$ & $0.2639(19)$ & $0.3850(20)$ & $2.6(9)$ \\
\hline $\mathrm{C} 22 \mathrm{~A}$ & $0.4912(16)$ & $0.3137(17)$ & $0.3828(17)$ & $1.3(8)$ \\
\hline $\mathrm{C} 23 \mathrm{~A}$ & $0.4281(16)$ & $0.2984(18)$ & $0.3496(18)$ & $2.0(9)$ \\
\hline $\mathrm{C} 24 \mathrm{~A}$ & $0.4147(16)$ & $0.2289(17)$ & $0.3310(20)$ & $2.7(10)$ \\
\hline $\mathrm{C} 25 \mathrm{~A}$ & $0.4685(18)$ & $0.1773(20)$ & $0.3378(21)$ & $3.9(11)$ \\
\hline $\mathrm{C} 26 \mathrm{~A}$ & $0.5252(16)$ & $0.1955(18)$ & $0.3667(19)$ & $2.1(9)$ \\
\hline C31A & $0.8121(23)$ & $0.1789(22)$ & $0.327(3)$ & $7.0(13)$ \\
\hline $\mathrm{C} 32 \mathrm{~A}$ & $0.7718(24)$ & $0.163(3)$ & $0.268(3)$ & $7.6(15)$ \\
\hline C33A & $0.800(3)$ & $0.097(3)$ & $0.242(3)$ & $11.5(20)$ \\
\hline C34A & $0.8581(23)$ & $0.0652(23)$ & $0.268(3)$ & $6.4(14)$ \\
\hline C35A & $0.8873(21)$ & $0.0874(23)$ & $0.3324(24)$ & $5.9(13)$ \\
\hline $\mathrm{C} 36 \mathrm{~A}$ & $0.8687(18)$ & $0.1534(20)$ & $0.3591(20)$ & $3.8(10)$ \\
\hline C41A & $0.8703(17)$ & $0.3141(18)$ & $0.3648(20)$ & $3.0(9)$ \\
\hline $\mathrm{C} 42 \mathrm{~A}$ & $0.8903(21)$ & $0.3469(22)$ & $0.4220(23)$ & $5.2(11)$ \\
\hline $\mathrm{C} 43 \mathrm{~A}$ & $0.9628(25)$ & $0.386(3)$ & $0.411(3)$ & $8.1(16)$ \\
\hline C44A & $0.9919(24)$ & $0.3900(23)$ & $0.3486(22)$ & $5.9(13)$ \\
\hline $\mathrm{C} 45 \mathrm{~A}$ & $0.9606(23)$ & $0.357(3)$ & $0.294(3)$ & $8.2(16)$ \\
\hline C46A & $0.8951(17)$ & $0.3182(18)$ & $0.2929(19)$ & $2.8(10)$ \\
\hline C51A & $0.6384(17)$ & $0.0440(18)$ & $0.4539(19)$ & $2.2(9)$ \\
\hline $\mathrm{C} 52 \mathrm{~A}$ & $0.5786(19)$ & $0.0141(20)$ & $0.4695(21)$ & $3.4(11)$ \\
\hline C53A & $0.5331(23)$ & $-0.0006(25)$ & $0.414(3)$ & $6.7(14)$ \\
\hline C54A & $0.5610(21)$ & $0.0010(24)$ & $0.3394(25)$ & $5.7(13)$ \\
\hline C55A & $0.6227(21)$ & $0.0213(23)$ & $0.329(3)$ & $6.2(13)$ \\
\hline C56A & $0.6586(19)$ & $0.0451(20)$ & $0.3885(22)$ & $3.7(11)$ \\
\hline
\end{tabular}

methyl or phenylthiomethyl group, which could occur in the chair conformation. A Dreiding model of (triphos) $\mathrm{Cr}(\mathrm{CO})_{3}$ revealed that each of the six-membered chelate rings in this octahedral complex must have the boat conformation. Thus in the formation of (triphos) $\operatorname{Cr}(\mathrm{CO})_{3}$, the energy gained from the coordination of the third phosphorus donor in triphos to the metal center must overcome that required for the formation of unfavored boat conformations, while it is apparently impossible for the sulfur donor in tripodal ligand 3 to do so, even though chelating sulfur ligands could form stable group VI metal carbonyl complexes [10]. To sum up, the 
tripodal ligand 3 behaves as a bidentate only in the coordination of group VI metal carbonyl complexes.

\section{Experimental}

Proton magnetic resonance spectra were recorded on Varian EM-390, a Bruker AC-E 200 or a Bruker AM-300WB spectrometers. Proton-decoupled phosphorus-31 NMR spectra were determined on Bruker AM-300WB or Bruker AC-E 200 spectrometers at $121.49 \mathrm{MHz}$ and $81.01 \mathrm{MHz}$, respectively. Chemical shifts are given in parts per million (ppm) relative to $85 \% \mathrm{H}_{3} \mathrm{PO}_{1}$ for ${ }^{31} \mathrm{P} \mathrm{NMR}$ spectra in $\mathrm{CDCl}_{3}$, unless otherwise noted. Chemical shifts upfield of the standard are regarded as negative.

Infrared spectra were recorded on a Perkin-Elmer 1310 or $983 \mathrm{G}$ instrument. Elemental analyses were carried out by use of a Perkin-Elmer $240 \mathrm{C}$ instrument. Melting points were determined on a Gallenkamp melting point apparatus and are uncorrected.

All of the reactions, manipulations, and purification steps involving phosphines were performed under dry nitrogen or argon. Air-sensitive liquids were transferred by Teflon flexineedles by use of nitrogen pressure or by syringe. The solutions were concentrated by a rotory evaporator with water aspirator pressure.

Tetrahydrofuran and diethyl ether were distilled under nitrogen from benzophenone ketyl. Toluene and xylene were distilled from sodium metal under nitrogen. Acetone was dried over 4 A molecular sieves. Other solvents and chemicals from commercial sources were used without further purification, except as noted.

5-(Phenylthiomethyl)-2,2,5-trimethyl-1,3-dioxane (7). Freshly cut sodium metal $(4.90 \mathrm{~g}, 0.213 \mathrm{~mol})$ was added to absolute ethanol $(150 \mathrm{ml})$ at ice-bath temperature. After all the sodium had disappeared, thiophenol $(25 \mathrm{ml}, 27.9 \mathrm{~g}, 0.245 \mathrm{~mol})$ was added and the solution was stirred for $0.5 \mathrm{~h}$. Tosylate $6(27.5 \mathrm{~g}, 87.5 \mathrm{mmol})$ was then added to the solution and the mixture was heated under reflux for $16 \mathrm{~h}$. Water ( 70 $\mathrm{ml}$ ) was then added and ethanol was removed by rotory evaporator. The residue was extracted with dichloromethane $(30 \mathrm{ml} \times 4)$ and the extract was dried and concentrated. The residue was distilled to give the desired product 7 as a clear, colorless liquid $\left(19.35 \mathrm{~g}, 88 \%\right.$ ): b.p. $106-107^{\circ} \mathrm{C} / 0.1-0.2$ Torr; ${ }^{1} \mathrm{H}$ NMR $\delta 7.43-7.14$ (m, 5 H), $3.71(\mathrm{~d}, J 11.7 \mathrm{~Hz}, 2 \mathrm{H}), 3.69(\mathrm{~d}, J 11.7 \mathrm{~Hz}, 2 \mathrm{H}), 3.19(\mathrm{~s}, 2 \mathrm{H}), 1.42(\mathrm{~s}, 3 \mathrm{H})$, $1.40(\mathrm{~s}, 3 \mathrm{H}), 0.91(\mathrm{~s}, 3 \mathrm{H})$.

Anal. Found: C, 66.63; H, 8.03. $\mathrm{C}_{14} \mathrm{H}_{20} \mathrm{O}_{2} \mathrm{~S}$ calc: C, 66.63; H. $7.99 \%$.

2-(Phenylthiomethyl)-2-methyl-1,3-propanediol (8). A mixture of 7 (3.74 g, 14.84 $\mathrm{mmol})$, ethanol $(5.5 \mathrm{ml})$, and water $(1.1 \mathrm{ml})$ containing a few drops of hydrochloric acid, was heated under reflux for $2 \mathrm{~h}$. The solution was concentrated and the residue was extracted with dichloromethane $(50 \mathrm{ml} \times 3)$. The extracts were dried and concentrated to give crude 8 as a white solid $(2.94 \mathrm{~g}, 93 \%)$. Recrystallization from methanol and hexane gave $\mathbf{8}$ as white crystals: m.p. $97-98^{\circ} \mathrm{C}$; IR $\left(\mathrm{CDCl}_{3}\right) 3505$ (br) $\mathrm{cm}{ }^{1} ;{ }^{1} \mathrm{H}$ NMR $\delta 7.44-7.18(\mathrm{~m}, 5 \mathrm{H}), 3.66(\mathrm{~s}, 4 \mathrm{H}), 3.13(\mathrm{~s}, 2 \mathrm{H}), 2.06(\mathrm{~s}, 2 \mathrm{H})$, $0.92(\mathrm{~s}, 3 \mathrm{H})$.

Anal. Found: $\mathrm{C}, 62.32 ; \mathrm{H}, 7.70 . \mathrm{C}_{11} \mathrm{H}_{16} \mathrm{O}_{2} \mathrm{~S}$ calc: $\mathrm{C}, 62.23 ; \mathrm{H}, 7.60 \%$.

2,2-Bis(methanesulfonyloxymethyl)-1-phenylthiopropane (9). To a solution of diol $8(3.72 \mathrm{~g}, 17.6 \mathrm{mmol})$ and triethylamine $(7.6 \mathrm{ml})$ in dichloromethane $(60 \mathrm{ml})$ was added mesyl chloride $(3.4 \mathrm{ml}, 5.03 \mathrm{~g}, 43.9 \mathrm{mmol})$ at $0^{\circ} \mathrm{C}$. The resulting solution was 
allowed to warm to room temperature and stirred for $2 \mathrm{~h}$. The reaction mixture was washed with a $4 \%$ aqueous sodium hydroxide solution, $10 \%$ hydrochloric acid, saturated sodium bicarbonate solution and brine. The organic portion was dried and concentrated. The residue was chromatographed on silica gel by elution with dichloromethane and ethyl acetate. A solvent mixture of $20 \%$ ethyl acetate in dichloromethane eluted the desired mesylate 9 as a viscous, colorless liquid (5.68 $\mathrm{g}$, 88\%): ${ }^{1} \mathrm{H}$ NMR $\delta 7.50-7.17(\mathrm{~m}, 5 \mathrm{H}), 4.10(\mathrm{~s}, 4 \mathrm{H}), 3.03(\mathrm{~s}, 2 \mathrm{H}), 2.97(\mathrm{~s}, 6 \mathrm{H}), 1.11$ $(\mathrm{s}, 3 \mathrm{H})$.

Anal. Found: $\mathrm{C}, 42.40 ; \mathrm{H}, 5.55 . \mathrm{C}_{13} \mathrm{H}_{20} \mathrm{O}_{6} \mathrm{~S}_{3}$ calc: $\mathrm{C}, 42.38 ; \mathrm{H}, 5.47 \%$.

2,2-Bis(diphenylphosphinomethyl)-1-phenylthiopropane (3). To a flask charged with triphenylphosphine $(6.75 \mathrm{~g}, 25.7 \mathrm{mmol})$ in THF $(100 \mathrm{ml})$ was added freshly cut lithium metal $(0.89 \mathrm{~g}, 127 \mathrm{mmol})$. This suspension was stirred at room temperature overnight. The excess lithium was removed by filtration under nitrogen. The filtrate was treated with t-butyl chloride $(3.05 \mathrm{ml}, 2.58 \mathrm{~g}, 28.0 \mathrm{mmol})$ at $0^{\circ} \mathrm{C}$ with stirring for $2 \mathrm{~h}$ to destroy phenylhithium. Mesylate $9(2.23 \mathrm{~g}, 6.05 \mathrm{mmol})$ in THF $(10 \mathrm{ml})$ was then added to the above solution. The resulting solution was heated to reflux for 4 h. Degassed water $(30 \mathrm{ml})$ was added. The organic layer was separated and the aqueous layer was extracted with ether $(30 \mathrm{ml} \times 2)$. All organic extracts were combined, dried, and concentrated. The residue was chromatographed on silica gel by elution with a mixture of hexane and dichloromethane. The fraction eluted by hexane $/ \mathrm{CH}_{2} \mathrm{Cl}_{2}$ (V/V: 2/3) gave the desired phosphine 3 as a clear, viscous liquid (1.88 g, 57\%): ${ }^{1} \mathrm{H}$ NMR $\delta 7.42-7.09(\mathrm{~m}, 25 \mathrm{H}), 3.19(\mathrm{~s}, 2 \mathrm{H}), 2.48(\mathrm{~d}, J(\mathrm{P}-\mathrm{C}-\mathrm{H}) 2.9$ $\mathrm{Hz}, 4 \mathrm{H}), 1.05$ (s, $3 \mathrm{H}) ;{ }^{31} \mathrm{P}$ NMR $\delta-25.57$.

Anal. Found: $\mathrm{C}, 76.66 ; \mathrm{H}, 6.24 . \mathrm{C}_{35} \mathrm{H}_{34} \mathrm{P}_{2} \mathrm{~S}$ calc.: $\mathrm{C}, 76.62 ; \mathrm{H}, 6.25 \%$.

General procedures for preparation of complexes $\mathbf{I O a}-10 \mathrm{c}$. In a flask equipped with reflux condenser were placed tripodal ligand $\mathbf{3}$ and equimolar amount of a metal carbonyl in an aromatic solvent [toluene for $\mathrm{Mo}(\mathrm{CO})_{6}$, xylene for $\mathrm{Cr}(\mathrm{CO})_{6}$ and $\left.\mathrm{W}(\mathrm{CO})_{6}\right]$. The mixture was heated to reflux under nitrogen. The progress of the reaction was monitored by IR spectroscopy in the range of metal carbonyl absorptions. The crude reaction mixture was then chromatographed on silica gel by elution with a mixture of hexane, dichloromethane and ethyl acetate. The fraction of the desired products was collected and recrystallized from ethyl acetate and hexane. The physical properties, spectroscopic and analytical data are listed in Table 1.

Crystallographic analysis. Crystals of $10 \mathrm{~b}$ were grown by evaporation from a solvent mixture of hexane and ethyl acetate. Measurement of cell dimensions and intensity data collections were carried out on an Enraf-Nonius CAD-4 diffractometer. Empirical formula X-ray data: $\mathrm{MoP}_{2} \mathrm{SC}_{39} \mathrm{O}_{4}, F w=722.37$. Crystal size $0.20 \times$ $0.20 \times 0.30 \mathrm{~mm}^{3}$. Space group; orthorhombic, $P b c 2_{1}$. Cell parameters (from the least-squares analysis of 24 reflections): $a=20.044(4), b=19.215(5), c=18.586(4)$ $\AA, V=7158(3) \AA^{3}, T=298 \mathrm{~K}, Z=8, D_{\mathrm{x}}=1.40 \mathrm{Mg} \mathrm{m}^{-3}, \mathrm{~F}(000)=2703, \mu=0.53$ $\mathrm{mm}^{-1}, \lambda\left(\mathrm{Mo}-K_{\alpha}\right)=0.7093 \AA$. The intensity data were collected in the $\omega-2 \theta$ scan mode. $2 \theta \max =44.9^{\circ}, 0 \leq h \leq 21,0 \leq k \leq 20,0 \leq l \leq 20$. A total of 4842 unique reflections were measured, of which 1680 were considered observed, i.e. $I>2.5 \sigma(I)$. Absorption corrections $(\psi)$ were made. Transmission factors were $0.919-0.999$.

The structure was solved by heavy atom method. Two independent molecules were found and there was no symmetry relation between them. The last least-squares cycle was calculated with 94 atoms (two independent molecules were found), 417 parameters. The atoms of Mo, $\mathrm{P}$ and $\mathrm{S}$ were refined anisotropically; all $\mathrm{C}$ and $\mathrm{O}$ 
(without hydrogen atoms) were refined isotropically. Refinement details are as follows, where $F_{\mathrm{o}}$ and $F_{\mathrm{c}}$ are the observed and calculated structure factor amplitudes, respectively; the function minimized was $\sum w\left(F_{\mathrm{o}}-F_{\mathrm{c}}\right)^{2}$, where $w=1 / \sigma^{2}\left(F_{\mathrm{o}}\right)$, $\sigma\left(F_{0}\right)$ from counting statistics; $R_{\mathrm{f}}=\Sigma\left|F_{\mathrm{o}}-F_{\mathrm{c}}\right| / \sum\left(F_{\mathrm{o}}\right) ; R_{\mathrm{w}}=\left[\sum\left(w\left(F_{\mathrm{o}}-F_{\mathrm{c}}\right)^{2}\right) /\right.$ $\left.\sum w\left(F_{o}\right)^{2}\right]^{1 / 2} ; S=\left[\sum\left(w\left(F_{o}-F_{c}\right)^{2}\right) /(\text { No. of reflections }- \text { No. of params })\right]^{1 / 2}$. For significant reflections, $R_{\mathrm{f}}=0.076, R_{\mathrm{w}}=0.068, S=2.506 ;(\Delta / \sigma)_{\max }=0.385$, peak in final map $-0.520 \sim 0.490 \mathrm{eA}^{-3}$. Atomic scattering factors were taken from International Tables for X-ray Crystallography [11]. Computing program: NRCC SDP VAX Package [12].

The atomic coordinates and thermal parameters are given in Table 4 . Iists of thermal parameters and structures factors are available from the authors.

\section{Acknowledgement}

Support of this work by the National Science Council NSC77-0208-M002-49 is acknowledged.

\section{References}

1 For a recent review on tripodal polytertiary phosphines see: L. Sacconi and F. Mani, in G.A. Melson and B.N. Figgis (Eds.), Transition Metal Chemistry, Vol. 8. Marcel Decker, New York. 1982, p. 179.

2 C. Bianchini, D. Masi. C. Mealli, A. Meli, M. Sabat, F. Vizza, Inorg. Chem, 27 (1988) 3716: C. Bianchini, D. Masi, A. Meli, M. Peruzzini, F. Zanobini. J. Am. Chem. Soc., 110 (1988) 6411; J.R. Bleeke, M.K. Hays, R.J. Wittenbrink, Organometallics, 7 (1988) 1417; B.S. McGilligan, L.M. Venanzi, M. Wolfer, Organometallies, 6 (1987) 946; G.V. Gocden, J.C. Huffman. K.G. Caulton. Inorg. Chem., 25 (1986) 2484; M.D. Vaira, M. Peruzzini, P. Stoppioni, Polyhedron, 5 (1986) 945; P. Peringer, M. Lusser, Inorg. Chim. Acta, 117 (1986) L25; S.C. Abrahams, A.P. Ginsberg. T.F. Koetzle. P. Marsh, C.R. Sprinkle, Inorg. Chem., 25 (1986) 2500; S.I. Hommeltoft. A.D. Cameron, I.A. Shackleton, M.E. Fraser, S. Fortier, M.C. Baird, Organometallics, 5 (1986) 1380; O.J. Scherer, R. Walter, W.S. Sheldrick, Angew. Chem. Int. Ed. Engl., 24 (1985) 525: F. Cecconi, C.A. Ghilardi, S. Midollini, S. Moneti, A. Orlandini, M. Bacci, J. Chem. Soc., Chem. Commun., (1985) 731; J. Rimmelin, P. Lemoine, M. Gross, A.A. Bahsoun, J.A. Osborn, Nouv, J. Chim.. 9 (1985) 181; D.J. Darensbourg. D.J. Zalewski, Organometallics, 4 (1985) 92; P. Janser. L.M. Venanzi, F. Bachechi, J. Organomet. Chem., 296 (1985) 229; R.B. King, P.N. Kapoor, R.N. Kapoor, lnorg. Chem., 10 (1971) 1841 ; J. Chatt, F.A. Hart, H.R. Watson, J. Chem. Soc.. (1962) 490: R. Davis, J.E. Fergusson. Inorg. Chim. Acta, $4(1970) 23$

3. S.-T. Liu, C.-H. Yieh, H.-J. Lu, Phosphorus and Sulfur, 37 (1989) 0000.

4 A.A. Ismail, I.S. Butler, J. Organomet, Chem, 346 (1988) 185.

5 S.I. Al-Resayes, S.I. Klein, H.W. Kroto, M.F. Meidine, J.F. Nixon, J. Chem. Soc., Chem. Commun.. (1983) 930.

6 V.W. Gash, J. Org. Chem., 37 (1972) 2197.

7 P.E. Garrou, Chem. Rev., 81 (1981) 229.

8 J.R. Gollogly, C.J. Hawkins, Inorg. Chem., 11 (1972) 156.

9 M.R. Churchill, A.L. Rheingold. R.L. Keiter, Inorg. Chem., 20 (1981) 2730.

10 S.G. Murray, F.R. Hartiey, Chem. Rev., 81 (1981) 365.

11 International Tables for X-ray Crystallography, Vol. IV Kynoch Press, Birmingham. 1974.

12 E.J. Gabe. F.L. Lee, Acta Cryst., A37 (1981) S339. 